\title{
HAZARDS TO LABORATORY STAFF IN CENTRIFUGING SCREW-CAPPED CONTAINERS
}

\author{
BY \\ F. WHITWELL, P. J. TAYLOR, AND A. J. OLIVER \\ From the Department of Pathology, Aintree Hospital, Liverpool
}

(RECEIVED FOR PUBLICATION DECEMBER 16, 1956)

\begin{abstract}
Reports on infectious diseases acquired by bacteriological laboratory staff from their work emphasize the risk of contracting tuberculosis by handling tuberculous specimens.

Recent advances in the chemotherapy of tuberculosis have greatly increased the amount of bacteriology done in routine hospital laboratories, primary culture and then sensitivity tests being required where previously a direct positive smear of sputum was considered an adequate investigation.
\end{abstract}

General protective measures, such as immunization of staff (who must be well trained in protective disciplines) and the provision of adequate accommodation, are obvious safeguards, but equally important is the re-examination of commonly used techniques to find out whether these are associated with any special risks.

Quantitative studies made with air samplers have shown that there is considerable dispersal of bacteria into the air during many routine bacteriological operations (Wedum, 1953: Reitman and Wedum, 1956), and an outcome of this work has been the elimination of avoidable risky methods and the provision of screens and exhaust fans or safety cabinets to reduce unavoidable risks.

These workers have studied the degree of air contamination during the technical operations concerned with centrifugation. They have shown that there is some aerial contamination during the filling of centrifuge tubes with cultures or infected material, during the removal of plugs or caps from centrifuge tubes, and when supernatant fluid is decanted from spun tubes. The atmosphere and apparatus are grossly contaminated when a tube breaks while being spun, but they consider that no risk is present during the normal running of a centrifuge. These findings have recently been summarized in an annotation in the Lancet (1956). which says "centrifuging a culture is safe enough -until the tube breaks."

The present investigation was started because it was noticed that less than three months after the laboratory wall had been painted glossy white there were parallel horizontal dirty streaks about 7 in. above the bench on the wall behind an angle-centrifuge, and on the side of an incubator close to the centrifuge (Fig. 1). This centrifuge is used only for concentrating homogenized sputa for tubercle culture, and it is in use for about three hours each day. There is a similar centrifuge in the biochemistry department, mainly used for spinning urines, and we found similar streaks on the wall behind this apparatus.

Both centrifuges have heads which take four 1 oz. "universal" containers, and these widenecked bottles are always used for spinning sputa and urines, with their screw-on tops, fitted with rubber washers, tightly secured. The centrifuge heads are fitted with screw-on tightly fitting metal covers, and these are always used.

It was decided to investigate the possibility that the contents of the universal containers were being sprayed around the laboratory during centrifuging, and the results prove that this has been happening

\section{Methods}

The method used for homogenizing and concentrating sputa proved to be of considerable importance in producing spray from the centrifuge. It is a Petroff method, modified in the following manner.

Four per cent. caustic soda is added to the sputum in a "universal" container. and after the screw-cap has



FIG. 1.-Bacteriological centrifuge in normal position showing the marks (faintly visible in this photograph) on the wall and side of the incubator about 7 in. above the bench top. 
been secured it is shaken intermittently during incubation at $37^{\circ} \mathrm{C}$., which lasts for about 20 minutes. The screw-cap is then removed and the alkali is neutralized with $10 \%$ hydrochloric acid added from a burette, using phenol red as the indicator. The final volume of fluid in the container is usually about $20 \mathrm{ml}$. This modification was evolved to reduce the time that the soda is in contact with the tubercle bacilli, to prevent their destruction by soda when specimens have to wait their turn for the centrifuge, and to get a better neutralization end-point by having a reasonable volume of fluid for the titration.

\section{Tests}

All tests, except for a few done on "swing-out" centrifuges, have been performed on the bacteriology angle-centrifuge (shown in Fig. 1) kept in its original position. Test fluids used have been suspensions of Staphylococcus albus and Chromobacterium prodigiosum, and $10 \%$ hydrochloric acid. In each test the volume of fluid in each container has been $15 \mathrm{ml}$. and four containers were spun.

At first all test fluids were treated in a manner designed to reproduce the manipulations of sputum concentration. The fluid was shaken in a tightly screwed universal container for about 10 seconds, and was then given a short incubation. Next, the cap was unscrewed and $5 \mathrm{ml}$. of water was added from a burette, after which the cap was screwed on again and the container was spun for 30 minutes at 3,500 r.p.m. After a few tests the method was modified by omitting incubation, unscrewing and rescrewing the cap without adding water from a burette, and spinning for only two minutes.

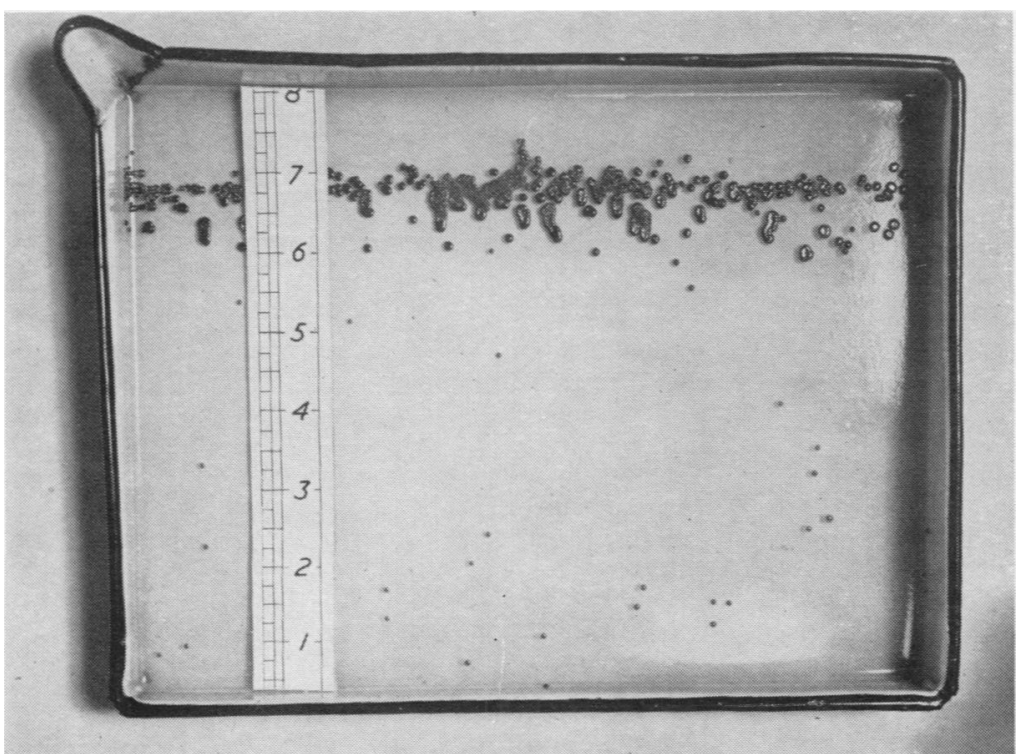

FIG. 2.-Mannite agar plate after 24 hours' incubation, showing a broad line of colonies of Chromobacterium prodigiosum at a level 7 in. above the bench top.
Staphylococcus albus Tests.-Filled " universal" containers were put in the centrifuge, and six agar plates were attached to the wall facing the centrifuge at different levels. The plates were exposed while the centrifuge ran at 3,500 r.p.m. for 30 minutes, and then were covered and incubated at $37^{\circ} \mathrm{C}$. for 24 hours.

Result.-Confluent colonies of staphylococci in horizontal bands were present in those plates which had been placed over the streaky marks on the wall. The other plates contained only a few colonies.

Chromobacterium prodigiosum Tests.-The centrifuge was loaded with filled containers and a large dish of mannite agar was placed against the wall, one edge resting on the bench-top. The centrifuge was run for two minutes, then the dish was covered and incubated at room temperature $\left(22^{\circ}\right.$ C.) for 24 hours.

Result.-A broad horizontal band of confluent Chromobacterium prodigiosum colonies grew on the agar, corresponding in position to the streaks on the wall 7 in. above the bench (Fig. 2).

During this test the air in front of the centrifuge was tested with a slit-sampler, but only one colony of Chromobacterium prodigiosum grew on a twominute slit-sampler plate. This result was surprising because droplets of suspension fell on the hands and face of the slit-sampler operator. The probable explanation is that the strong air current created by the centrifuge directed the spray right over the intake slit of the sampler.

This test was repeated three times in a modified form, the centrifuge being run for 30 seconds and stopped by friction in 30 seconds. The slit-sampler was placed three feet away from the centrifuge, and ran for two minutes starting at the same time as the centrifuge. An average of 13 colonies per plate was obtained on these three runs, but the friction applied to the centrifuge reduces the values of these figures.

The centrifuge test was repeated on another occasion after the room had been well ventilated. Mannite plates were exposed on the benches, at varying distances from the centrifuge, and Chromobacterium prodigiosum colonies grew on the plates placed up to 52 in. away from the centrifuge.

Hydrochloric Acid Tests.-A less laborious test, and one more quickly read, consists of filling the containers with $10^{\circ}$ o hydrochloric acid and using filter-paper soaked in Andrade's indicator and then dried to detect the spray, which shows on the paper as red dots. 
(a) A test similar to the bacteriological tests was done, and it give the same result.

(b) This test was repeated, but a new piece of test paper was inserted each 30 seconds. This showed that when the centrifuge was started slowly most of the spray occurred in the first 60 seconds, but in the first 30 seconds when started fast. There was some fine spray between 60 and 120 seconds, but none later. considerable "fall-out" in the area below the lower stream, and a small amount beyond it.

These tests show that there is gross leakage of fluid? into the atmosphere from "universal" containers spun in the centrifuge.

Next, possible leakage into the centrifuge buckets $\frac{\overparen{\Phi}}{\Phi}$ and over the outsides of the "universal " containers was tested.

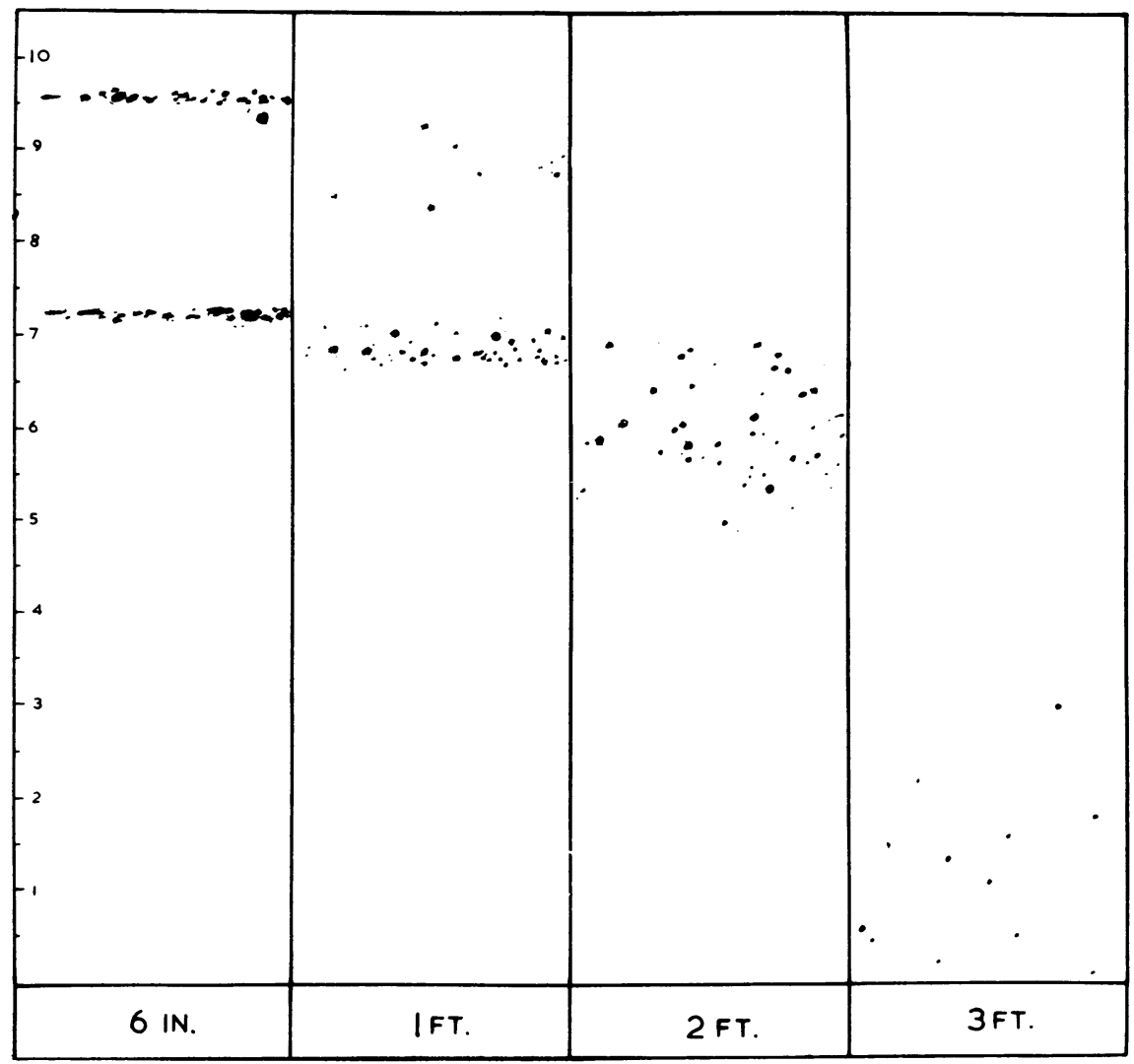

FIG. 3.-Copies of the test papers held at various distances from the centrifuge, showing acid spray markings at different levels above the surface of the bench.

(c) The centrifuge part of the test was repeated many times, and on each occasion the test paper was held at a different distance from the centrifuge. The results (Fig. 3) have enabled us to plot the main course of the spray from the centrifuge, and this is shown in Fig. 4. The spray leaves the centrifuge in two streams. The upper stream probably leaves from the junction of the metal cover with the centrifuge head, and it passes outwards horizontally for about 16 in., when it becomes impossible to trace. The lower stream is the main one, starting at the lower rim of the centrifuge head, and extending horizontally for over two feet when it starts to fall and reaches bench-level at about 42 in. As the spray gets further out it becomes finer and more widely distributed. Apart from the main channels of spray shown in Fig. 4, there is (d) Filled containers were washed in water and dried, and test papers were attached to their sides ${ }^{-r}$ with "sellotape." Centrifuge buckets and rubber cushions were washed and dried. The containers N were put in their buckets and spun for two minutes. $\sigma$ The test papers then showed that acid had leaked out into the buckets and over the surfaces of the con- $\frac{0}{E}$ tainers.

(e) The same procedure as in $(d)$ was followed, but the containers were spun at 3,000 r.p.m. in a "swingout" type of centrifuge for two minutes. The test papers then showed more extensive leakage than in $\overrightarrow{\mathbb{D}}$ the angle-centrifuge test. This test was repeated with two other "swing-out" centrifuges, and always gave the same result. 


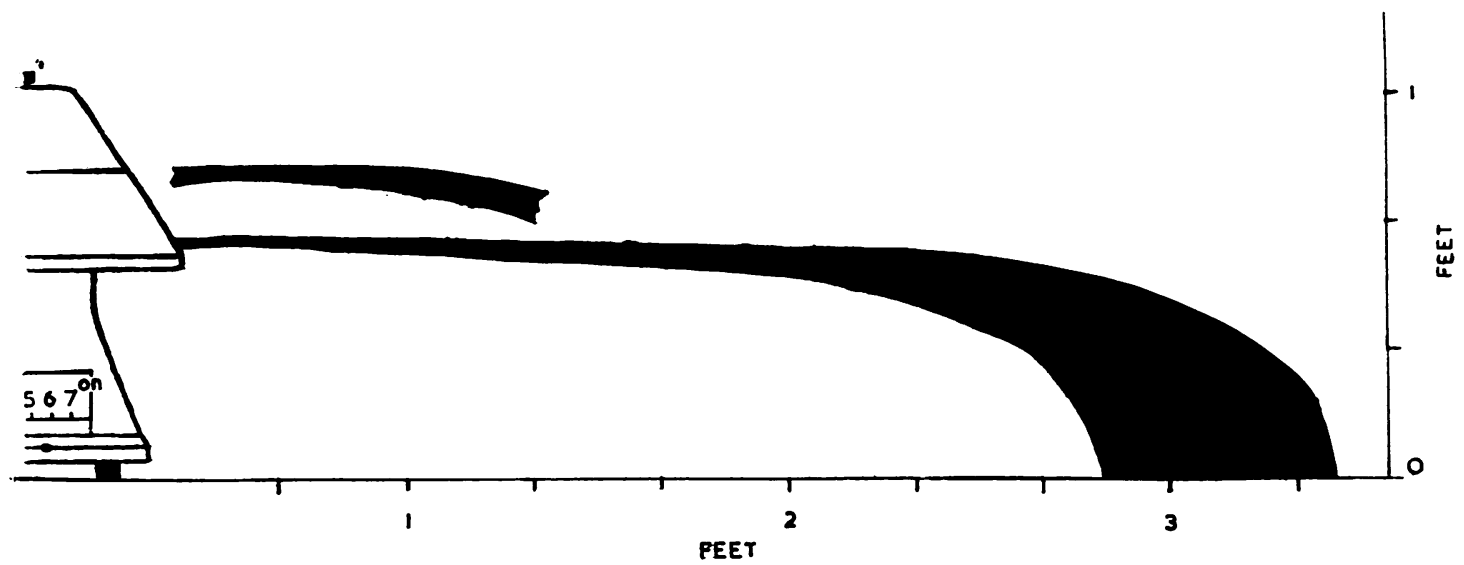

FIG. 4.-Scale diagram showing the main streams of spray from the angle-centrifuge (represented by black areas).

We thought that the spray probably came from fluid trapped in the head of the containers, between the glass and metal threads. This was found to be so.

When the test fluid was put into the containers with pipettes so that no fluid touched the head and neck of the container and a dry cap was then securely screwed on, no spraying occurred during spinning even after vigorous shaking. The occasional exceptions to this result were due to defective rubber washers or metal caps. When a test container, which had been treated as in the earlier tests, was opened before spinning, had its head and neck flamed, and was fitted with a new dry cap, there was no spraying on spinning. Also there was no spraying when a container which had been carefully filled with a pipette was spun without any screw-cap.

\section{Discussion}

Often when infected fluid in a " universal " container is spun in an angle-centrifuge some of the material is sprayed over a wide area around the centrifuge, the size of this area being about $7 \mathrm{ft}$. in diameter. At the same time the material contaminates the buckets and the outsides of the containers.

If the containers are spun in a "swing-out" type of centrifuge the buckets and outsides of the containers may be heavily contaminated, though neither the inside of the centrifuge casing nor the atmosphere is contaminated.

The cause of the contamination is fluid trapped between the threads of the bottle and the cap. This may happen through careless filling of the container, or it may be caused by uncapping and recapping a container which has been shaken or inverted, for during this operation moisture from the surface of the rubber washer nearly always spreads over the metal thread of the cap and becomes lodged between the metal and glass thread on recapping.
Uncapping and recapping before centrifugation of "universal" containers holding infected material is a common bacteriological operation. Sometimes it is done in concentrating sputum, as in our technique, but more often it is done in a later stage to wash the bacterial deposit before culture or inoculation.

Universal containers have many advantages over test-tubes and centrifuge tubes, so our technique is being modified to retain the use of these containers but to eliminate the risk of spraying. Two methods are being tried, the first one involving neutralization after spinning, so that no uncapping and recapping takes place before spinning. The second method will involve decanting the neutralized sputum into a fresh "universal" container and fitting it with a new screw-cap before spinning. In addition to these measures, the centrifuge has been surrounded with a perspex casing.

\section{Summary}

Fluid can escape from sealed "universal" containers during centrifugation, because it has become trapped in the thread of the screw-caps. When spun in an angle-centrifuge this fluid forms a spray which extends over an area of about $7 \mathrm{ft}$. diameter, and it contaminates the outside of the container and the bucket. When spun in a "swing-out" centrifuge there is no spray, but the bucket with its container become contaminated.

Methods of avoiding this contamination are discussed.

We wish to thank Mr. G. Wilkins, of the Department of Radiology, Aintree Hospital, for the illustrations.

\section{REFERENCES}

Annotation (1956). Lancet, 2, 880.

Reitman, M., and Wedum, A. G. (1956). Publ. Hlth Rep. (Wash.), 71, 659 .

Wedum, A. G. (1953). Amer. J. publ. Hlth, 43, 1428. 